\title{
Thermodynamical transcription of density functional theory with minimum Fisher information
}

\author{
Á. Nagy \\ Department of Theoretical Physics, \\ University of Debrecen, \\ H-4002 Debrecen, Hungary \\ e-mail:anagy@phys.unideb.hu
}

(Dated: February 1, 2018)

\begin{abstract}
Ghosh, Berkowitz and Parr designed a thermodynamical transcription of the ground-state density functional theory and introduced a local temperature that varies from point to point. The theory, however, is not unique because the kinetic energy density is not uniquely defined. Here we derive the expression of the phase-space Fisher information in the GBP theory taking the inverse temperature as the Fisher parameter. It is proved that this Fisher information takes its minimum for the case of constant temperature. This result is consistent with the recently proven theorem that the phase-space Shannon information entropy attains its maximum at constant temperature.
\end{abstract}




\section{INTRODUCTION}

The role of Fisher information [1] in quantum mechanics and density functional theory was first emphasized by Sears, Parr and Dinur [2] more than twenty years ago. They studied the relationship between the quantum mechanical kinetic energy and the Fisher information.

Later, using the principle of extreme physical information the Schrödinger equation [3-5], the Euler equation [6] and the Kohn-Sham equations [7] were derived. Fisher information has been applied among others in studying atoms and molecules [8-21].

The ground-state density functional theory was reformalized as 'thermodynamics' by Ghosh, Berkowitz and Parr [22]. The phase-space distribution function maximizing the phase-space Shannon information entropy was derived. They obtained a local MaxwellBoltzmann distribution function and introduced the concept of the local temperature. The theory has several applications and extensions [23-43]. Among others, the local thermodynamic formalism was extended to ensembles of excited states [48] and ensemble local temperature was defined.

The Ghosh-Berkowitz-Parr theory is not unique, because one can apply several expressions for the kinetic energy density. Therefore, the local temperature is not unique either. The ambiguity of the local kinetic energy and temperature was addressed by several authors [44-47]. In [46] the N-particle quasiprobability distribution maximising the Shannon limit of the Tsallis entropy was proposed, though it was not worked through. It has recently been proved [49] that it is possible selecting the kinetic energy density so that the local temperature be a constant for the whole system under consideration. In this case the kinetic energy density is proportional to the electron density and the temperature is proportional to the kinetic energy. Furthermore, the kinetic energy density corresponding to the constant temperature, maximizes the Shannon information entropy. A similar result has been obtained for the ensemble extension of the theory [50].

Here we derive the expression of the Fisher information in the Ghosh-Berkowitz-Parr theory taking the inverse temperature for the Fisher parameter. Then we prove that this Fisher information takes its minimum for the case of constant temperature. That is, we arrive at the remarkable result that at constant temperature the phase-space Shannon information entropy takes its maximum and the phase-space Fisher information has its minimum. These simple and interesting theorems give a deeper insight into the Ghosh-Berkowitz-Parr theory. 
The following section provides the summary the Ghosh-Berkowitz-Parr theory. Section III. presents the new theory with the Fisher information. The last section is devoted to examples and discussion.

\section{THERMODYNAMICAL TRANSCRIPTION OF DENSITY FUNCTIONAL THEORY}

In this section the "thermodynamical" transcription [22] is summarized. In the density functional theory we study a system of $N$ electrons moving in a local external potential $v(\mathbf{r})$. According to the Hohenherg-Kohn theorems [51] the ground-state electron density $n(\mathbf{r})$ determines $v(\mathbf{r})$ up to a trivial additive constant and the ground-state total energy takes its minimum at the true density. Moreover, there exists a non-interacting, Kohn-Sham system, where the electrons move independently in a common, local Kohn-Sham potential.

Introduce a phase-space distribution function $f(\mathbf{r}, \mathbf{p})$ with the properties:

$$
\begin{aligned}
& \int d \mathbf{p} f(\mathbf{r}, \mathbf{p})=n(\mathbf{r}), \\
& \int d \mathbf{r} n(\mathbf{r})=N
\end{aligned}
$$

and

$$
\int d \mathbf{p} \frac{p^{2}}{2 m} f(\mathbf{r}, \mathbf{p})=t_{s}(\mathbf{r})
$$

$m$ is the electron mass. The non-interacting kinetic energy density $t_{s}(\mathbf{r})$ integrates to the non-interacting kinetic energy $E_{k i n}$ :

$$
E_{k i n}=\int d \mathbf{r} t_{s}(\mathbf{r})
$$

The marginal conditions (1-3) are satisfied by a number of distribution functions. Note that in the GBP theory it is not required that the phase-space distribution function integrates to the momentum density $[52,53]$.

Take the distribution function that maximizes the Shannon information entropy

$$
\begin{gathered}
S=\int d \mathbf{r} s(\mathbf{r}) \\
s(\mathbf{r})=-k \int d \mathbf{p} f(\ln f-1)
\end{gathered}
$$


subject to the constraints above, that is, the correct density (Eq. (1)) and the correct noninteracting kinetic energy ( Eq. (3)) are fixed. $k$ is the Boltzmann constant. The maximizing distribution function is a local Maxwell-Boltzmann type function:

$$
f(\mathbf{r}, \mathbf{p})=e^{-\alpha(\mathbf{r})} e^{-\beta(\mathbf{r}) p^{2} / 2 m} .
$$

$\alpha(\mathbf{r})$ and $\beta(\mathbf{r})$ are $\mathbf{r}$-dependent Lagrange multipliers. Substituting the distribution function (7) into the constraint (3) we are led to

$$
t_{s}(\mathbf{r})=\frac{3}{2} \frac{n(\mathbf{r})}{\beta(\mathbf{r})}
$$

Introducing the local temperature $T(\mathbf{r})$ with the definition

$$
\beta(\mathbf{r})=\frac{1}{k T(\mathbf{r})},
$$

the non-interacting kinetic energy density takes the form of the ideal gas expression:

$$
t_{s}(\mathbf{r})=\frac{3}{2} n(\mathbf{r}) k T(\mathbf{r})
$$

and the distribution function can be rewritten as

$$
f(\mathbf{r}, \mathbf{p})=[2 \pi m k T(\mathbf{r})]^{-3 / 2} n(\mathbf{r}) e^{-p^{2} / 2 m k T(\mathbf{r})} .
$$

The local temperature is expressed with the kinetic energy density and can vary from point to point. It is important to emphasize that the local temperature is not uniquely defined as the kinetic energy density is not unique. Adding a term that integrates to zero to the kinetic energy density results another kinetic energy density with the same kinetic energy but different local temperature. Usually the gradient form of the kinetic energy density is applied, because it is everywhere positive, though any form resulting the kinetic energy can do.

\section{FISHER INFORMATION}

The Fisher information [1] measures the information that we can obtain for the parameter $\theta$ of the distribution function $g(x \mid \theta)$. It is defined as

$$
I_{g}(\theta)=\int \frac{\left[\frac{\partial g(x \mid \theta)}{\partial \theta}\right]^{2}}{g(x \mid \theta)} d x .
$$


Consider now the phase-space distribution function normalized to 1:

$$
\varrho(\mathbf{r}, \mathbf{p})=\frac{1}{N} f(\mathbf{r}, \mathbf{p})=\frac{1}{N}\left[\frac{\beta(\mathbf{r})}{2 \pi m}\right]^{3 / 2} n(\mathbf{r}) e^{-\beta(\mathbf{r}) p^{2} / 2 m}
$$

and take $\beta$ for the parameter $\theta$ of the distribution function. The phase-space Fisher information is now defined as

$$
I(\beta)=\int \frac{\left[\frac{\partial \varrho(\mathbf{r}, \mathbf{p} \mid \beta)}{\partial \theta}\right]^{2}}{\varrho(\mathbf{r}, \mathbf{p} \mid \beta)} d \mathbf{r} d \mathbf{p} .
$$

Note that it is a generalization of the original definition as $\beta$ in the GBP theory is a function of r. Substituting Eq. (13) into Eq.(14) and integrating for the momentum, the phase-space Fisher information takes the form

$$
I(\beta)=\frac{3}{2 N} \int \frac{n(\mathbf{r})}{(\beta(\mathbf{r}))^{2}} d \mathbf{r} .
$$

We can express the phase-space Fisher information with the non-interacting kinetic energy density $t_{s}$ instead of $\beta$ :

$$
I\left(t_{s}\right)=\frac{2}{3 N} \int \frac{\left(t_{s}(\mathbf{r})\right)^{2}}{n(\mathbf{r})} d \mathbf{r}
$$

Now, we minimize the Fisher information (16) under the condition that the kinetic energy $E_{k i n}$ is fixed:

$$
\tilde{I}=\frac{2}{3 N} \int \frac{\left(t_{s}(\mathbf{r})\right)^{2}}{n(\mathbf{r})} d \mathbf{r}+\xi\left(E_{k i n}-\int t_{s}(\mathbf{r}) d \mathbf{r}\right) .
$$

$\xi$ is the Lagrange multiplier. Note that a given ground-state Kohn-Sham problem is considered, therefore the density $\mathrm{n}(\mathbf{r})$ is fixed. The variation leads to

$$
\frac{4}{3 N} \frac{t_{s}}{n}-\xi=0
$$

Using Eqs. (9) and (10) it can be rewritten as

$$
\beta=\frac{2}{N \xi}
$$

As the Lagrange multiplier $\xi$ is a constant, the inverse temperature $\beta$ (and the temperature T) is also constant.

Because of Eq. (4) the value of the temperature is given by

$$
\frac{1}{\beta}=k T=\frac{2}{3} E_{k i n}
$$


That is, the kinetic energy density for which the Fisher information is minimum gives constant temperature. Furthermore, the kinetic energy density is proportional to the electron density and the temperature is proportional to the kinetic energy. Previously, we found that the maximum of the phase-space Shannon information entropy was also obtained for constant temperature [49]. It is remarkable that both the minimum phase-space Fisher information and the maximum phase-space Shannon information entropy are attained at constant temperature.

\section{DISCUSSION AND ILLUSTRATIVE EXAMPLES}

It is important to emphasize that the Fisher information studied here is different from the ones applied previously in atomic and molecular physics. First, this is a phase-space Fisher information, that is, the distribution function depends on the phase-space variables. Secondly, here the Fisher information with a parameter taken as the inverse temperarure is considered. In the great majority of the applications the parameter is the position. The fluctuation of the observed position from the true position is translationally invariant $g(x \mid \theta)=g(x-\theta)$. Therefore, the Fisher information (12) can be rewritten as

$$
I=\int \frac{\left[\frac{\partial g(x)}{\partial x}\right]^{2}}{g(x)} d x
$$

and it does not depend on the parameter $\theta[4]$. In our case, however, the parameter is the inverse temperature $\beta$ of the phase-space distribution function and the phase-space Fisher information parameter does depend on $\beta$.

Here a special form of the Ghosh-Berkowitz-Parr theory has been explored. The one for which the phase-space Fisher information is minimum, The corresponding phase-space distribution function is the Maxwell-Boltzmann function with constant temperature (Eq. (11)). We would like to stress that the nonuniqueness of the original Ghosh-Berkowitz-Parr theory has advantages: one can select a kinetic energy density and local temperature which are suitable for the investigation of a given problem, the ones that are more appropriate for studying properties including structure, bonding, spectroscopy reactivity and other characteristics.

Let the first example be the linear harmonic oscillator. The ground-state density is given 
by

$$
n(x)=\left(\frac{m \omega}{\pi \hbar}\right)^{1 / 2} e^{-m \omega x^{2} / \hbar}
$$

where $m$ and $\omega$ are the mass and the frequency, respectively. From the ground-state kinetic energy

$$
E_{k i n}=\frac{1}{4} \hbar \omega
$$

one can immediately obtain the temperature

$$
k T=\frac{1}{\beta}=\frac{\hbar \omega}{2} .
$$

In this example we have a one-dimensional system, therefore Eq. (8) should be modified as

$$
t(x)=\frac{1}{2} k \operatorname{Tn}(x)=\frac{n(x)}{2 \beta} .
$$

For the Fisher information we obtain

$$
I(\beta)=\frac{1}{2} \int \frac{n(\mathbf{r})}{(\beta(\mathbf{r}))^{2}} d \mathbf{r}
$$

When the temperature is constant the Fisher information takes its minimum:

$$
I=\frac{1}{8}(\hbar \omega)^{2}
$$

Take the Hydrogen atom in its ground state as a second example. The density is given by

$$
n(r)=\frac{1}{\pi}\left(\frac{Z}{a_{0}}\right)^{3} e^{-2 Z r / a_{0}}
$$

where

$$
a_{0}=\frac{\hbar^{2}}{m e^{2}}
$$

and $Z, r, m$ and $e$ are the atomic number, the radial distance, the electron mass and the magnitude of the electronic charge, respectively. From the well-known form of the groundstate kinetic energy

$$
E_{k i n}=\frac{\hbar^{2} Z^{2}}{2 m a_{0}^{2}},
$$


one can immediately obtain the temperature

$$
k T=\frac{1}{\beta}=\frac{Z^{2} m e^{4}}{3 \hbar^{2}} .
$$

The minimum Fisher information can be written as

$$
I(\beta)=\frac{3}{2} \int \frac{n(\mathbf{r})}{(\beta(\mathbf{r}))^{2}} d \mathbf{r} .
$$

When the temperature is constant the Fisher information takes its minimum:

$$
I=\frac{3}{2 \beta^{2}}=\frac{1}{6} \frac{Z^{4} m^{2} e^{4}}{\hbar^{4}}
$$

In summary, we derived the expression of the Fisher information in the Ghosh-BerkowitzParr theory having the inverse temperature as the Fisher parameter. The result is not unique because of the ambiguity of the kinetic energy density. The minimum of the phase-space Fisher information was searched over the kinetic energy densities with fixed kinetic energy and density. We arrived at the remarkable result that the phase-space Fisher information takes its minimum at constant temperature. This result is in harmony with our previous theorem that the phase-space Shannon information entropy takes its maximum at constant temperature. The kinetic energy density is found to be proportional to the electron density and the temperature proportional to the kinetic energy.

\section{Acknowledgments}

This research was supported by the EU-funded Hungarian grant EFOP-3.6.2-16-201700005 and the National Research, Development and Innovation Fund of Hungary, financed under 123988 funding scheme.

[1] R. A. Fisher, Proc. Cambridge Philos. Soc., 22 ( 1925) 700.

[2] S. B. Sears, R. G. Parr, U. Dinur, Isr. J. Chem. 19 ( 1980) 165.

[3] B. R. Frieden, Am. J. Phys. 57 (1989) 1004.

[4] B. R. Frieden, Physics form Fisher Information. A Unification,(Cambridge University Press, Cambridge, 1998.

[5] M. Reginatto, Phys. Rev. A 58 (1998) 1775. 
[6] Á. Nagy, J. Chem. Phys. 119 (2003) 1199401.

[7] R. Nalewajski, Chem. Phys. Lett. 372 ( 2003) 28.

[8] E. Romera, P. Sánchez-Moreno, J. S. Dehesa, Chem. Phys. Lett. 414 (2005) 468.

[9] Á. Nagy, Chem. Phys. Lett. 425 (2006) 154.

[10] Á. Nagy, K. D. Sen, Phys. Lett. A 360 (2006) 291.

[11] I. Hornyák, Á. Nagy, Chem. Phys. Lett. 120 (2007) 132.

[12] E. Romera, J. S. Dehesa, J. Chem. Phys. 120 (2004) 8906.

[13] E. Romera, Mol. Phys. 100 (2002) 3325.

[14] S. B. Liu, J. Chem. Phys. 126 (2007) 191107.

[15] Á. Nagy, Chem. Phys. Lett. 449 (2007) 212.

[16] Á. Nagy, S. B. Liu, Phys. Lett. A 372 (2008) 1654.

[17] J. B. Szabó, K. D. Sen, Á. Nagy, Phys. Lett. A 372 (2008) 2428.

[18] R. F. Nalewajski, Found. Chem. 16 (2014) 27.

[19] R. F. Nalewajski, R. G. Parr, J. Phys. Chem. A 105 (2001) 7391.

[20] R. F. Nalewajski, Chem. Phys. Lett. 372 (2003), 28.

[21] P. W. Ayers, Theor. Chem. Acc. 115, (2006) 370.

[22] S.K. Ghosh, M. Berkowitz, R.G. Parr, Proc. Natl. Acad. Sci. USA 81 (1984) 8028.

[23] S. K. Ghosh, R. G. Parr, Phys.Rev. A 34 (1986) 785.

[24] R. G Parr, K. Rupnik, S. K. Ghosh, Phys. Rev. Lett. 56 (1986) 1555.

[25] C. Lee, R. G. Parr, Phys. Rev. A 35 (1987) 2377.

[26] S. R. Gadre, R. D. Bendale, Int. J. Quant. Chem. 28 (1985) 311.

[27] S. R. Gadre, S. B. Sears, S. J. Chakravorty, R. D. Bendale, Phys. Rev. A 32 (1985) 2602.

[28] S. R. Gadre, Phys. Rev. A 30 (1984) 620.

[29] S. R .Gadre, S. A. Kulkani, I. H. Shrivastava, Chem. Phys. Lett. 166 (1990) 445.

[30] S. R.Gadre, R. D. Bendale, S. P. Gejii, Chem. Phys. Lett. 117 (1985) 138.

[31] Á. Nagy, R. G. Parr, Proc. of Ind. Acad. Sci. (Chem. Sci) 106 (1984) 217.

[32] Á. Nagy, R. G. Parr, J. Mol. Struct. (Theochem) 501 (2000) 101.

[33] Á. Nagy, R. G. Parr, Int. J. Quantum Chem. 58 (1996), 323.

[34] Á. Nagy, Proc. Ind. Acad. Sci. (Chem. Sci) 106 (1994) 251.

[35] Á. Nagy, in Reviews of Modern Quantum Chemistry, ed. K. D. Sen (World Scientific, 2002) Vol.I, p.413. 
[36] Á. Nagy, J. Mol. Struct Theochem 943 (2010) 48.

[37] A. A. Astakhov, A. I. Stash, V. G. Tsirelson, Int. J. Quant. Chem. 116 (2016) 237.

[38] P. Planchard, J. M. Gracia-Bondia and J. C. Varilly, Int. J. Quant. Chem. 112 (2012) 1134.

[39] C. Y. Rong, T. Lu, P. W. Ayers, P. K. Chattaraj, S. B. Liu, Phys. Chem. Chem.Phys. 17 (2015), 4977.

[40] C. Y. Rong, T. Lu, P. K. Chattaraj, S. B. Liu, Indian J. Chem. A 53 (2014) 970.

[41] S. B. Liu, C. Y. Rong, Z. M. Wu, Acta Phys. Chim, Sin. 31 (2015 2057.

[42] P. W. Ayers and S. Jenkins, Comp. Theor. Chem, 1053 (2015) 112.

[43] S. B. Liu, Acta Phys. Chim, Sin. 32 (2016) 98.

[44] L. Cohen, J. Chem. Phys. 70 (1979) 788.

[45] L. Cohen, J. Chem. Phys. 80 (1984) 4277.

[46] P. W. Ayers, R. G. Parr, Á. Nagy, Int. J. Quantum Chem, 90 (2002) 309.

[47] J. S. Anderson, P. W. Ayers and J. I. R. Hernandez, J. Phys. Chem, 114 (2010) 8884.

[48] Á. Nagy, Indian J. Chem. A 53 (2014) 965.

[49] Á. Nagy, Int. J. Quantum Chem. 117 (2017) e5396. doi: 10.1002/qua.25396

[50] Á. Nagy, Acta Phys. Chim. Sin. (2017) doi: 10.3866/PKU.WHXB201709221

[51] P. Hohenberg, W. Kohn, Phys. Rev. 136 (1964) B 864.

[52] L. Cohen, J. Math. Phys. 7 (1966) 781.

[53] L. Cohen, Phil. of Science 33 (1966) 317. 